\title{
PRODUÇÃO IN VITRO DE EMBRIÕES UTILIZANDO-SE SÊMEN SEXADO DE TOUROS 5/8 GIROLANDO
}

\section{PRODUCTION OF IN VITRO EMBRYO USING SEXED SPERM OF 5/8 GIROLANDO BULLS}

\author{
Pábola Santos Nascimento ${ }^{1^{*}}$ \\ Maiana Silva Chaves ${ }^{1}$ \\ Antônio Santana dos Santos Filho ${ }^{2}$ \\ Sebastião Inocêncio Guido² \\ Maria Madalena Pessoa Guerra ${ }^{1}$ \\ Cláudio Coutinho Bartolomeu ${ }^{1}$
}

1 Universidade Federal Rural de Pernambuco, Departamento de Medicina Veterinária, Recife, PE, Brasil.

2Instituto Agronômico de Pernambuco, Recife, PE, Brasil.

*Autor para correspondência - pabolasn@hotmail.com

\section{Resumo}

Avaliou-se a taxa de produção de blastocisto "in vitro" utilizando-se o sêmen bovino sexado. Foram utilizados três reprodutores para verificar a variação individual do sêmen, taxas de clivagem e produção embrionária. $\mathrm{O}$ trabalho utilizou-se de biotécnicas reprodutivas, análise computadorizada do sêmen pós-descongelação e sondas fluorescentes para análises de integridade da célula espermática (membrana plasmática, membrana acrossomal e potencial mitocondrial). Um total de 959 oócitos passou por etapas de maturação in vitro, fertilização in vitro (sexado, $\mathrm{n}=473$; convencional, $\mathrm{n}=486$ ) e cultivo in vitro. A taxa de clivagem foi observada no D2 e a de blastocistos no D7. Os dados foram analisados pelo programa SPSS 16.0 empregando-se a análise de variância (ANOVA), sendo o teste t-Student usado para detectar diferenças entre os grupos e o Qui-quadrado para análise dos resultados da produção in vitro $(\mathrm{P}<0,05)$. Os resultados diferiram entre o sêmen convencional $(31,06 \%)$ e sexado $(21,10 \%)$ para produção de blastocisto. Quando comparada a produção de blastocisto individualmente nas amostras de sêmen sexado (27,69\%; $17,93 \%$ e $25,56 \%$, touros 1,2 e 3, respectivamente), percebeu-se que T2 < T1 e T1=T3 e T2=T3. Quanto às análises de cinética espermática, as amostras de sêmen sexado mostraram diferenças entre os touros nas variáveis velocidade curvilínea, velocidade linear e velocidade do trajeto em que o $\mathrm{T} 1(117,7 \pm 1,6 \mu \mathrm{m} / \mathrm{s} ; 60,0 \pm 0,3 \mu \mathrm{m} / \mathrm{s} ; 73,6 \pm 0,4 \mu \mathrm{m} / \mathrm{s}$, respectivamente) quando comparado aos touros T2 $(80,2 \pm 2,3 \mu \mathrm{m} / \mathrm{s} ; 47,0 \pm 2,0 \mu \mathrm{m} / \mathrm{s} ; 57,7 \pm 0,9 \mu \mathrm{m} / \mathrm{s}$, respectivamente) e $\mathrm{T} 3(86,4 \pm 5,7 \mu \mathrm{m} / \mathrm{s}$; $46,2 \pm 2,7 \mu \mathrm{m} / \mathrm{s} ; 53,8 \pm 2,8 \mu \mathrm{m} / \mathrm{s}$, respectivamente) obteve valores mais elevados. As análises da integridade da célula espermática não diferiram entre as amostras de sêmen convencional, já no sêmen sexado a integridade de membrana foi a variável que diferiu estatisticamente entre os touros, em que o T1 $(38 \pm 2,7)$ diferiu do T3(53,8 $\pm 1,8)(\mathrm{P}=0,009)$, mas não divergiu do T2 $(44,1 \pm 4,4)$. É possível concluir que o sêmen sexado foi menos eficiente na produção de blastocisto quando 
comparado ao sêmen convencional. As análises de cinética e de integridade foram compatíveis com o potencial fertilizante das amostras de sêmen em touros da raça 5/8 Girolando.

Palavras-chave: bovino; CASA; FIV.

\begin{abstract}
We evaluated the "in vitro" blastocyst rate production using bovine sexed semen. Semen from three bulls was used to verify the individual's semen variation, cleavage rates and embryo production. In this study, we employed reproductive biotechnologies, computer analysis of post-thawed semen and fluorescent probes for sperm cells integrity analysis (plasma membrane, acrosome membrane and mitochondrial potential). A total of 959 oocysts went through in vitro maturation steps for in vitro fertilization and cultivation, being 473 with sexed semen and 486 with conventional semen. The cleavage rate was observed in blastocysts on D2 and D7. Data were analyzed by SPSS 16.0 software using analysis of variance (ANOVA), Student's t-test was used to detect differences between groups, and chi-square analysis for in vitro production results $(\mathrm{P}<0.05)$. The results differed between conventional (31.06\%) and sexed semen (21.10\%) in the obtainment of blastocyst. When the blastocyst production was individually compared in sexed semen samples $(27.69 \%$, $17.93 \%$ and $25.56 \%$, bulls 1,2 and 3, respectively) we verified $\mathrm{T} 2<\mathrm{T} 1$ and $\mathrm{T} 1=\mathrm{T} 3$ and $\mathrm{T} 2=\mathrm{T} 3$. As for sperm kinetic analyzes, the sexed semen samples showed differences among bulls in curvilinear speed, linear speed and path velocity variables where $\mathrm{T} 1(117.7 \pm 1.6 \mu \mathrm{m} / \mathrm{s} ; 60.0 \pm 0.3$ $\mu \mathrm{m} / \mathrm{s}, 73.6 \pm 0.4 \mu \mathrm{m} / \mathrm{s}$, respectively) showed the highest values when compared to T2 bulls (80.2 \pm $2.3 \mu \mathrm{m} / \mathrm{s}, 47.0 \pm 2.0 \mu \mathrm{m} / \mathrm{s}, 57.7 \pm 0,9 \mu \mathrm{m} / \mathrm{s}$, respectively) and T3 $(86.4 \pm 5.7 \mu \mathrm{m} / \mathrm{s}, 46.2 \pm 2.7 \mu \mathrm{m} / \mathrm{s}$, $53.8 \pm 2.8 \mu \mathrm{m} / \mathrm{s}$, respectively). Analyses of sperm cell integrity did not differ among conventional semen samples, but in the sexed semen, membrane integrity was the variable that differ statistically among bulls once $\mathrm{T} 1(38 \pm 2.7)$ differed from $\mathrm{T} 3(538 \pm 1.8)(\mathrm{P}=0.009)$ but did not differ from $\mathrm{T} 2$ $(44.1 \pm 4.4)$. It is possible to conclude that sexed semen was less efficient in blastocyst production when compared with conventional semen. Analyses of kinetic and integrity were consistent with the fertilization potential of semen samples from 5/8 Girolando bulls.
\end{abstract}

Keywords: bovine, CASA, FIV.

Recebido em: 07 outubro 2014

Aceito em: 27 abril 2015

\title{
Introdução
}

A raça Girolando tem apresentado crescimento significativo, colaborando na formação e desenvolvimento de novas bacias leiteiras, que estão embasadas na lucratividade da atividade, cujo objetivo primordial é alcançar a máxima produção de leite/vaca/dia ${ }^{(1)}$. Caso a concepção seja atrasada, a ineficiência reprodutiva pode levar à redução na produção de leite, comprometendo economicamente a atividade. Dessa forma, a literatura na área de biotecnologia reprodutiva desenvolvida com a raça Girolando (pura sintética, com grau de sangue 5/8 e outros) merece incremento e enfoque pelo papel importante na pecuária nacional.

A fertilização in vitro (FIV), uma das técnicas mais avançadas das novas biotécnicas da reprodução animal, surgiu na tentativa de ampliar o potencial reprodutivo, resolvendo, em alguns casos, a 
própria infertilidade. No entanto, uma das dificuldades enfrentadas que precisam ser superadas nos estudos dessa técnica é a grande variação nos resultados na produção de blastocisto que pode ser resultado da interferência não só da doadora, mas também do sêmen utilizado ${ }^{(2)}$.

A sexagem do sêmen utilizado na FIV pode produzir embriões do sexo desejado em menor período de tempo, porém, o processo de sexagem debilita os espermatozoides, deixando-os mais sensíveis devido ao estresse químico e físico aos quais são expostos durante o processo, diminuindo sua capacidade de fecundação e consequentemente produzindo menores taxas de desenvolvimento embrionário, quando comparados aos não-sexados ${ }^{(3)}$. As razões específicas das baixas taxas de fertilidade do sêmen sexado não são totalmente conhecidas.

Desde 1940, cientistas reconhecem a necessidade de obter informações objetivas acerca da porcentagem de espermatozoides móveis (de um ejaculado/de uma palheta), por acreditarem que a obtenção de dados precisos sobre o movimento espermático poderia ser utilizada para prever o potencial de fertilidade de um macho ou selecionar o melhor procedimento para a preparação do sêmen $^{(4)}$. O potencial de fertilização de uma amostra de sêmen pode ser obtido pela análise das características seminais correlacionando os resultados com a técnica de FIV ${ }^{(5)}$. Vários estudos têm sido realizados com o objetivo de identificar esse potencial fecundante peculiar e a maioria dos resultados mostram diferenças entre os tipos de sêmen (sexado/convencional), raça, reprodutores utilizados, partidas e palhetas de um mesmo animal ${ }^{(6-8)}$.

A utilização de análises computadorizadas e sondas fluorescentes por meio de microscopia de epifluorescência ou citometria de fluxo são métodos que vêm sendo utilizados amplamente nos últimos anos em diversas espécies ${ }^{(9,10)}$, incluindo humanos ${ }^{(11)}$, pois estes procedimentos certificam com maior acurácia a qualidade do sêmen na tentativa de minimizar os efeitos da avaliação convencional $^{(12)}$.

Testes laboratoriais são utilizados como critério para excluir amostras de sêmen de baixo potencial fecundante ${ }^{(13)}$, no entanto, o grande desafio seria apontar, dentre as diversas variáveis obtidas pelo CASA (Computer-Assisted Semen Analysis), aquelas que teriam uma maior influência sobre o potencial de fertilidade ${ }^{(14)}$, e dentre os animais férteis, aqueles que suportariam os danos causados pela técnica de sexagem mantendo sua capacidade fecundante ${ }^{(3)}$. Sua adoção na rotina poderia ajudar no diagnóstico do que está atualmente classificado como infertilidade inexplicável ${ }^{(13)}$.

Diante do exposto, o objetivo deste trabalho foi comparar a taxa de blastocistos produzidos com sêmen sexado e convencional de três touros da raça 5/8 Girolando, a partir da sua utilização na fertilização in vitro de oócitos provenientes de ovários obtidos em abatedouros, associando-os às análises laboratoriais (CASA e sondas).

\section{Material e Métodos}

O experimento foi realizado entre os meses de julho a dezembro de 2013, na Estação Experimental do Instituto Agronômico de Pernambuco (IPA), localizada no município de Arcoverde, que está localizado a uma latitude $08^{\circ} 25^{\prime} 08^{\prime \prime}$ sul, longitude $37^{\circ} 03^{\prime} 14^{\prime \prime}$ oeste, a uma altitude de 663 metros, no sertão pernambucano, clima semiárido, precipitação pluviométrica anual de 694,2 mm e uma temperatura média anual de $23,75^{\circ} \mathrm{C}$. As análises do sêmen foram realizadas no Laboratório de Andrologia do Departamento de Medicina Veterinária - ANDROLAB, da Universidade Federal Rural de Pernambuco/Recife. 
Os ovários bovinos foram coletados no matadouro dos municípios de Arcoverde e Pedra, imediatamente após o abate, e transportados em garrafa térmica contendo solução fisiológica a $0,9 \%$ e $30 \mathrm{mg} / \mathrm{mL}$ de sulfato de gentamicina, à temperatura de $37{ }^{\circ} \mathrm{C}$, para o Laboratório de Reprodução e Melhoramento Genético Animal do Instituto Agronômico de Pernambuco (IPA).

No laboratório, os ovários foram lavados de 2 a 3 vezes com solução salina fosfatada e tamponada PBS (pH 7.0-7.2) e colocados em banho maria à $37^{\circ} \mathrm{C}$. Os folículos entre 3 a $8 \mathrm{~mm}$ de diâmetro foram aspirados com seringa de $10 \mathrm{~mL}$ e agulha hipodérmica 40x12 mm e o aspirado foi alocado em um tubo cônico de $50 \mathrm{~mL}$. Após 5 minutos de espera, para decantação, o sobrenadante foi desprezado e, posteriormente, foram adicionados $10 \mathrm{~mL}$ de PBS. O conteúdo foi colocado em uma placa de Petri com fundo riscado para facilitar a busca dos complexos cumulus- oócito (COC's), com auxílio de estereomicroscópio (SMZ-745, Nikon, Tóquio, Japão). Foram utilizados apenas os COC's com citoplasma homogêneo e pelo menos três camadas de células do cumulus compactas, classificados como grau 1, com base na classificação morfológica descrita por Gonçalves et al. ${ }^{(15)}$.

Anteriormente ao início do processo de produção in vitro, durante todas as etapas, os meios foram estabilizados em estufa com $5 \%$ de $\mathrm{CO}_{2}$ e atmosfera úmida à $38,5{ }^{\circ} \mathrm{C}$ por 24 horas. Os oócitos ( $n=959)$ foram lavados com meio TALP, divididos em grupos (15 estruturas/gota) e levados para maturação por 24 horas em estufa (WaterJacketed $\mathrm{CO}_{2}$ Incubators, Thermo Scientific Forma® Series II, USA/CANADA), com atmosfera saturada de umidade com $5 \%$ de $\mathrm{CO}_{2}$ a $38,5{ }^{\circ} \mathrm{C}$, em uma placa de Petri de $35 \mathrm{~mm}$ com 4 gotas de $100 \mu \mathrm{L}$ com meio TCM 199, cobertas com óleo mineral, segundo Camargo et. al. ${ }^{(16)}$.

Para a fertilização in vitro, o sêmen (palhetas com espermatozoides sexados de mesma partida; palhetas com espermatozoides não sexados/convencionais de mesma partida) congelado/descongelado dos mesmos touros (Descongelador de Sêmen WTA/Biodux, São Paulo, Brasil), à $37^{\circ} \mathrm{C}$ por 30 segundos, proveniente de três touros da raça 5/8 Girolando (CRV Lagoa Sertãozinho/SP), foi centrifugado a $6.000 \mathrm{~g}$ por $7 \mathrm{~min}$ (ESPRESSO Microcentrífuga pessoal, ThermoScientific, USA/Canada) e selecionado pelo método Gradiente descontinuo de Percoll (4590\%), para obtenção de espermatozoides móveis. As células foram ressuspendidas em 1,5 mL de meio FERT- TALP contendo heparina $(33 \mu \mathrm{g} / \mathrm{mL})$ e centrifugadas novamente com mesma velocidade e tempo. A fertilização in vitro foi realizada em gotas de $100 \mu \mathrm{L}$ de meio FERT- TALP contendo 15 oócitos desnudados parcialmente, completadas com uma concentração de 2,0 × $10^{5}$ espermatozoides/mL, cobertas com óleo mineral, segundo Camargo et. al. ${ }^{(16)}$. Espermatozoides e oócitos foram incubados por 18 horas em atmosfera saturada de umidade com $5 \%$ de $\mathrm{CO}_{2}$ à $38,5^{\circ} \mathrm{C}$. Os touros 1,2 e 3 passaram pelos mesmos procedimentos.

Os zigotos foram lavados em três gotas de $70 \mu \mathrm{L}$ do meio SOF, transferidos para placas com gotas de $100 \mu \mathrm{L}$ do mesmo meio, cobertas com óleo mineral, e foram encaminhadas à estufa com atmosfera saturada de umidade com $5 \%$ de $\mathrm{CO}_{2}$, à $38,5{ }^{\circ} \mathrm{C}$, para desenvolvimento. No terceiro dia, com 48 horas do início do cultivo, foi realizado o primeiro feeding (troca de $50 \%$ do meio de cultivo - SOF) e a avaliação da taxa de clivagem. No sétimo dia, foram observados os percentuais de blastocistos.

As mesmas palhetas de sêmen (sexado e convencional), de três touros da raça 5/8 Girolando, de igual partida, que foram utilizadas para a produção in vitro, foram usadas também para as análises de integridade da célula espermática, em todas as repetições do experimento. Foram feitas três repetições de cada procedimento, uma referente a cada repetição da produção in vitro.

Para a cinética, $5 \mu \mathrm{L}$ de cada amostra foram colocados em uma lâmina previamente aquecida 
$\left(37^{\circ} \mathrm{C}\right)$ e uma lamínula depositada sobre a gota. A lâmina foi avaliada em microscopia de contraste de fase (Eclipse 50i; Nikon, Tóquio, Japão) e as imagens foram captadas com uma câmera digital Basler A312FC (Basler Vision Technologies, Ahrensburg, Alemanha). As imagens foram capturadas e analisadas utilizando-se o software SCA ${ }^{\mathrm{TM}}$ v 5.1 (Microptics, SL, Barcelona, Espanha). Pelo menos cinco campos microscópicos, não consecutivos, selecionados aleatoriamente por amostra, foram digitalizados, registrando-se pelo menos 2.000 espermatozoides móveis. As definições de parâmetros padrão foram: linearidade (LIN), retilinearidade (STR), expressos em valores porcentuais (\%); velocidade curvilínea (VCL), velocidade linear progressiva (VSL) e velocidade média da trajetória (VAP), expressas em micrometros por segundo $(\mu \mathrm{m} / \mathrm{s})$; amplitude de deslocamento lateral da cabeça (ALH), expresso em micrometros $(\mu \mathrm{m})$; e frequência de batimento flagelar (BCF), expresso em hertz (Hz).

Integridade acrossômica, integridade da membrana plasmática e potencial de membrana mitocondrial foram avaliados por microscopia de epifluorescência (Carl Zeiss, Göttingen, Alemanha). Para a determinação da integridade do acrossoma, os espermatozoides foram corados com isotiocianato de fluoresceína conjugado a Peanutagglutinin (FITC-PNA), segundo Silva et al. ${ }^{(10)}$. Alíquotas $(10 \mu \mathrm{L})$ de sêmen foram utilizadas para preparação dos estiraços, os quais foram secos à temperatura ambiente. Para corar as células, uma alíquota de $30 \mu \mathrm{L}$ da solução de FITCPNA (100 $\mu \mathrm{g} / \mathrm{mL}$ em PBS) foi depositada sobre cada lâmina, incubada em câmara úmida a $4{ }^{\circ} \mathrm{C}$ por 20 min., lavada em PBS e seca no escuro. No momento da avaliação, $5 \mu \mathrm{L}$ de meio de montagem (4,5 mL glicerol, 0,5 mL PBS, $5 \mathrm{mg}$ azida sódica e $5 \mathrm{mg}$ p-phenylenediamine) foram depositados sobre a lâmina e coberto com lamínula. Um total de 200 espermatozoides por lâmina foi examinado, usando-se filtros LP 515-nm para emissão e 450 - 490 BP nm para excitação, em um microscópio de epifluorescencia (Carl Zeiss, Göttingen, Alemanha; 1000 X). Os espermatozoides foram classificados como portadores de acrossoma intacto, quando apresentavam a região acrossomal corada em verde fluorescente, ou de acrossoma reagido, quando apresentavam fluorescência apenas na região equatorial da cabeça ou quando não corados em verde fluorescente.

A integridade da membrana plasmática foi determinada empregando-se uma combinação de diacetato de carboxifluoresceína (CFDA) e iodeto de propídio (PI), tal como descrito por Silva et al. ${ }^{(17)}$. Alíquotas $(50 \mu \mathrm{L})$ de sêmen foram diluídas em $150 \mu \mathrm{L}$ de solução Tris, contendo $5 \mu \mathrm{L}$ de CFDA $(0,46 \mathrm{mg} / \mathrm{mL}$ em DMSO) e $20 \mu \mathrm{L}$ de PI $(0,5 \mathrm{mg} / \mathrm{mL}$ em PBS $)$, incubadas por $10 \mathrm{~min}$. à 37 ${ }^{\circ} \mathrm{C}$, e fixadas com PBS contendo 0,5\% de glutaraldeído. Usando-se PAD 485/20 nm de excitação e PAD 580-630 nm filtros de emissão, 200 células por lâmina foram examinados (400X). Fluorescências verdes e vermelhas foram interpretadas como membrana intacta e membrana danificada, respectivamente.

Para determinação do potencial da membrana mitocondrial, um método adaptado de Guthrie e Welch $^{(18)}$ foi utilizado. Alíquotas $(50 \mu \mathrm{L})$ de sêmen foram diluídas em $150 \mu \mathrm{L}$ de Tris, contendo 5 $\mu \mathrm{L}$ de fluorocromo catiônico lipofílico JC-1 (0,15 mM em DMSO) e incubadas durante 10 min, à $37{ }^{\circ} \mathrm{C}$, e fixadas com $0,5 \%$ de glutaraldeído. Duzentos espermatozoides por lâmina foram examinados (400X), utilizando-se LP $515 \mathrm{~nm}$ e emissão de 450-490 nm BP filtros de excitação. Os espermatozoides foram classificados como alto potencial de membrana mitocondrial, ao emitirem fluorescência laranja na peça intermediária, e baixo potencial de membrana mitocondrial, quando emitiram fluorescência verde.

Os dados foram analisados pelo programa estatístico SPSS 16.0, empregando-se a análise de variância (ANOVA). O teste t-Student foi usado para detectar diferenças entre os grupos e o Qui- 
quadrado, para análise dos resultados da produção in vitro. Para todas as análises, os valores foram considerados significativos $(\mathrm{P}<0,05)$.

Previamente às análises, dados percentuais para motilidade progressiva, integridade de acrossoma, integridade da membrana plasmática e potencial da membrana mitocondrial, linearidade, retilinearidade foram transformados em arcoseno. Os resultados foram expressos em média \pm erro padrão da média.

\section{Resultados}

Quando se comparou sêmen sexado com o convencional do mesmo touro, verificou-se que para o T3 houve menor clivagem com sêmen sexado, mas sem diferença na produção de blastocistos. Para o T2 não houve diferença na clivagem, mas foi diferente na produção de blastocistos. Para o T1 não houve diferença na clivagem ou na produção de blastocistos (Tabela 1).

Tabela 1: Comparação entre diferentes estádios de desenvolvimento de embriões produzidos in vitro com sêmen sexado e convencional de três touros da raça 5/8 Girolando

\begin{tabular}{cccccc}
\hline & TIPO & N & CLIVADOS & D7/Clivados & $\begin{array}{c}\text { Blastocistos } \\
\text { D7/oócitos }\end{array}$ \\
\hline & & & & & \\
& & & & & \\
T1 & Sex. & 195 & $119(61,02 \%)^{\mathrm{a}}$ & $54(45,3 \%)^{\mathrm{aD}}$ & $54(27,69 \%)^{\mathrm{aD}}$ \\
& Conv. & 207 & $141(68,11 \%)^{\mathrm{a}}$ & $61(43,2)^{\mathrm{a}}$ & $61(29,46 \%)^{\mathrm{a}}$ \\
& Total & 402 & & & \\
& Sex. & 145 & $84(57,93 \%)^{\mathrm{a}}$ & $26(30,9 \%)^{\mathrm{aD}}$ & $26(17,93 \%)^{\mathrm{aC}}$ \\
& Conv. & 134 & $90(67,16 \%)^{\mathrm{a}}$ & $41(45,5 \%)^{\mathrm{b}}$ & $41(30,59 \%)^{\mathrm{b}}$ \\
& Total & 279 & & $\mathrm{P}<0,05$ & $\mathrm{P}<0,05$ \\
& Sex. & 133 & $73(54,88 \%)^{\mathrm{a}}$ & $34(46,5 \%)^{\mathrm{aC}}$ & $34(25,56 \%)^{\mathrm{aCD}}$ \\
& Conv. & 145 & $105(72,41 \%)^{\mathrm{b}}$ & $49(46,6 \%)^{\mathrm{a}}$ & $49(33,79 \%)^{\mathrm{a}}$ \\
& Total & 278 & $\mathrm{P}<0,01$ & & $114(24,10 \%)^{\mathrm{a}}$ \\
& Sex. & 473 & $276(58,35 \%)^{\mathrm{a}}$ & $114(41,3 \%)^{\mathrm{a}}$ & 110 \\
& Conv. & 488 & $336(69,13 \%)^{\mathrm{b}}$ & $151(44,9 \%)^{\mathrm{a}}$ & $151(31,06 \%)^{\mathrm{b}}$ \\
& 959 & $\mathrm{P}<0,01$ & & $\mathrm{P}<0,05$ \\
\hline
\end{tabular}

Médias ( \pm SEM) com letras sobrescritas diferentes (a,b entre sêmens) ou (C,D entre touros) na mesma coluna são significativamente diferentes $(\mathrm{P}<0,05)$. T1, T2 e T3: touros.

O resultado estatístico evidenciou diferenças $(\mathrm{P}<0,05)$ nas análises do CASA entre os touros, tanto em comparações feitas entre as amostras de sêmen sexado quanto nas amostras de sêmen convencional, em diversas variáveis, principalmente as que se referem ao padrão de movimento dos espermatozoides VCL (Velocidade curvilínea); VSL (Velocidade linear); e VAP (Velocidade de trajeto). No sêmen convencional, os valores médios de VCL, VSL e VAP obtidos pelo T1 foram $66,5 \pm 0,8 \mu \mathrm{m} / \mathrm{s} ; 36,9 \pm 0,3 \mu \mathrm{m} / \mathrm{s}$ e $46,4 \pm 0,5 \mu \mathrm{m} / \mathrm{s}$, respectivamente, e pelo T2 foram $46,2 \pm 2,5 \mu \mathrm{m} / \mathrm{s}$; $27,6 \pm 2,3 \mu \mathrm{m} / \mathrm{s}$ e $33,8 \pm 2,4 \mu \mathrm{m} / \mathrm{s}$, respectivamente $(\mathrm{P}<0,05)$. Os valores obtidos pelo $\mathrm{T} 3$ foram 
$64,6 \pm 5,1 \mu \mathrm{m} / \mathrm{s} ; 35,3 \pm 0,6 \mu \mathrm{m} / \mathrm{s}$ e $44,2 \pm 1,0 \mu \mathrm{m} / \mathrm{s}$, respectivamente, e diferiram do $\mathrm{T} 2(\mathrm{P}<0,05)$ como pode ser observado na Tabela 2.

Os estudos realizados entre as amostras de sêmen sexado apresentaram diferenças entre os touros nas variáveis VCL, VSL e VAP, em que o T1 $(117,7 \pm 1,6 \mu \mathrm{m} / \mathrm{s} ; 60,0 \pm 0,3 \mu \mathrm{m} / \mathrm{s} ; 73,6 \pm 0,4 \mu \mathrm{m} / \mathrm{s}$, respectivamente), quando comparado aos touros T2 $(80,2 \pm 2,3 \mu \mathrm{m} / \mathrm{s} ; 47,0 \pm 2,0 \mu \mathrm{m} / \mathrm{s} ; 57,7 \pm 0,9 \mu \mathrm{m} / \mathrm{s}$, respectivamente) e T3 $(86,4 \pm 5,7 \mu \mathrm{m} / \mathrm{s} ; 46,2 \pm 2,7 \mu \mathrm{m} / \mathrm{s} ; 53,8 \pm 2,8 \mu \mathrm{m} / \mathrm{s}$, respectivamente), obteve valores mais elevados. Já para as variáveis LIN (Linearidade) e ALH (Deslocamento lateral da cabeça), não se observou diferença estatística. O resultado da BCF (frequência de batimento de cauda) foi significativo entre os touros, de modo que o T1 apresentou $14,5 \pm 0,3 \mathrm{~Hz}>\mathrm{T} 3(13,0 \pm 0,4$ $\mathrm{Hz})>\mathrm{T} 2(10,5 \pm 0,3 \mathrm{~Hz})$. Essas diferenças foram vistas também através dos valores de $\mathrm{P}=0,04$; $\mathrm{P}=0,009$, respectivamente.

Tabela 2: Análise comparativa do sêmen convencional, sexado e convencional x sexado de três touros $5 / 8$ Girolando quanto às variáveis da cinética espermática.

\begin{tabular}{cccccccc}
\hline & N & \multicolumn{2}{c}{ T1 } & \multicolumn{2}{c}{ T2 } & \multicolumn{2}{c}{ T3 } \\
\hline & & Convenc. & Sexado & Convenc. & Sexado & Convenc. & Sexado \\
\hline VCL & 3 & $66,5 \pm 0,8^{\mathrm{a}}$ & $117,7 \pm 1,6^{\mathrm{C}}$ & $46,2 \pm 2,5^{\mathrm{b}}$ & $80,2 \pm 2,3^{\mathrm{D}}$ & $64,6 \pm 5,1^{\mathrm{a}}$ & $86,4 \pm 5,7^{\mathrm{D}}$ \\
VSL & 3 & $36,9 \pm 0,3^{\mathrm{a}}$ & $60,0 \pm 0,3^{\mathrm{C}}$ & $27,6 \pm 2,3^{\mathrm{b}}$ & $47,0 \pm 2,0^{\mathrm{D}}$ & $35,3 \pm 0,6^{\mathrm{a}}$ & $46,2 \pm 2,7^{\mathrm{D}}$ \\
VAP & 3 & $46,4 \pm 0,5^{\mathrm{a}}$ & $73,6 \pm 0,4^{\mathrm{C}}$ & $33,8 \pm 2,4^{\mathrm{b}}$ & $57,7 \pm 0,9^{\mathrm{D}}$ & $44,2 \pm 1,0^{\mathrm{a}}$ & $53,8 \pm 2,8^{\mathrm{D}}$ \\
LIN & 3 & $55,4 \pm 1,0^{\mathrm{a}}$ & $55,7 \pm 0,7^{\mathrm{C}}$ & $59,6 \pm 2,4^{\mathrm{a}}$ & $58,7 \pm 2,4^{\mathrm{C}}$ & $55,1 \pm 3,1^{\mathrm{a}}$ & $53,6 \pm 2,6^{\mathrm{C}}$ \\
STR & 3 & $79,4 \pm 1,5^{\mathrm{a}}$ & $81,6 \pm 0^{\mathrm{C}}$ & $81,4 \pm 1,0^{\mathrm{a}}$ & $81,4 \pm 2,2^{\mathrm{C}}$ & $79,7 \pm 0,3^{\mathrm{a}}$ & $85,7 \pm 0,5^{\mathrm{C}}$ \\
ALH & 3 & $2,7 \pm 0,0^{\mathrm{a}}$ & $3,2 \pm 0,2^{\mathrm{C}}$ & $2,0 \pm 0,0^{\mathrm{a}}$ & $2,4 \pm 0,1^{\mathrm{C}}$ & $2,6 \pm 0,4^{\mathrm{a}}$ & $3,2 \pm 0,3^{\mathrm{C}}$ \\
BCF & 3 & $10,1 \pm 0,2^{\mathrm{a}}$ & $14,5 \pm 0,3^{\mathrm{C}}$ & $9,3 \pm 0,4^{\mathrm{a}}$ & $10,5 \pm 0,3^{\mathrm{D}}$ & $9,9 \pm 0,2^{\mathrm{a}}$ & $13,0 \pm 0,4^{\mathrm{E}}$ \\
\hline
\end{tabular}

VCL (Velocidade curvilínea, $\mu \mathrm{m} / \mathrm{s}$ ); VSL (Velocidade linear, $\mu \mathrm{m} / \mathrm{s}$ ); VAP (Velocidade de trajeto, $\mu \mathrm{m} / \mathrm{s}$ ); LIN (Linearidade, \%); STR (Retilineariade, \%); ALH (Deslocamento lateral da cabeça, $\mu \mathrm{m}$ ); BCF (Frequência de batimento de cauda, $\mathrm{Hz}$ ). Médias $( \pm \mathrm{SEM})$ com letras sobrescritas diferentes $(\mathrm{a}, \mathrm{b}$ entre sêmens) ou ( $C, D, E$ entre touros) na mesma linha são significativamente diferentes $(P<0,05)$.

Tabela 3: Médias e erros-padrão da integridade da célula espermática do sêmen convencional e sexado de três touros $5 / 8$ Girolando.

\begin{tabular}{|c|c|c|c|c|c|c|}
\hline \multicolumn{7}{|c|}{ Sondas Fluorescentes } \\
\hline & \multicolumn{2}{|c|}{ T1 } & \multicolumn{2}{|c|}{ T2 } & \multicolumn{2}{|c|}{ T3 } \\
\hline & Convenc. & Sexado & Convenc. & Sexado & Convenc. & Sexado \\
\hline $\begin{array}{c}\text { Membrana } \\
\text { citoplasmática }\end{array}$ & $47,5 \pm 11,8^{\mathrm{a}}$ & $38,0 \pm 2,7^{\mathrm{C}}$ & $40,1 \pm 7,3^{\mathrm{a}}$ & $44,1 \pm 4,4^{\mathrm{CD}}$ & $66,5 \pm 2,7^{\mathrm{a}}$ & $53,8 \pm 1,8^{\mathrm{D}}$ \\
\hline $\begin{array}{l}\text { Pot. de memb. } \\
\text { mitocondrial } \\
\text { Membrana }\end{array}$ & $66 \pm 19,0^{\mathrm{a}}$ & $79,6 \pm 4,8^{C}$ & $73,6 \pm 3,6^{a}$ & $81,0 \pm 3,2^{\mathrm{C}}$ & $82,6 \pm 7,9^{a}$ & $85,5 \pm 2,7^{\mathrm{C}}$ \\
\hline acrossomal & $71,8 \pm 4,0^{\mathrm{a}}$ & $56,0 \pm 12,8^{\mathrm{C}}$ & $74,2 \pm 3,0^{\mathrm{a}}$ & $55,8 \pm 10,5^{\mathrm{C}}$ & $76,6 \pm 0,4^{\mathrm{a}}$ & $69,3 \pm 1,1^{\mathrm{C}}$ \\
\hline
\end{tabular}

Médias $( \pm \mathrm{SEM})$ com letras sobrescritas diferentes (a,b entre sêmens) ou (C,D,E entre touros) na mesma linha são significativamente diferentes $(\mathrm{P}<0,05)$. 
Ao se compararem os resultados para as análises com sondas fluorescentes, integridade de membrana plasmática, integridade de acrossoma e potencial mitocondrial não foram observadas diferenças entre os grupos de sêmen convencional. Já para o sêmen sexado, a integridade de membrana foi a única variável que diferiu estatisticamente entre os touros (Tabela 3), em que o T1 $(38 \pm 2,7)$ diferiu do T3 $(53,8 \pm 1,8)(\mathrm{P}=0,009)$.

\section{Discussão}

Os resultados evidenciaram que a diferença entre sêmen sexado e convencional foi inerente ao touro, não havendo diferença entre touros para o sêmen convencional, no entanto, foram observadas diferenças quando da utilização do sêmen sexado, provavelmente em decorrência do sêmen de alguns touros ser mais sensível aos processos da sexagem por citometria de fluxo do que o de outros, tendo em vista que, na produção de blastocisto do sêmen sexado, T2 (17,93\%) apresentou resultados inferiores ao $\mathrm{T} 1(27,69 \%)$.

Os resultados apresentados, semelhante a dados da literatura ${ }^{(6-8)}$, evidenciaram o efeito inerente ao touro, no sêmen, sobre a fertilização in vitro de oócitos bovinos, apresentando diferenças significativas entre touros da mesma raça e em partidas do mesmo touro, tanto na taxa de fertilização quanto na taxa de sobrevivência embrionária, confirmando que o fator touro tem importância determinante quanto à capacidade de fertilização, bem como quanto à capacidade de crescimento dos embriões clivados até mórula e seu posterior desenvolvimento até o estádio de blastocisto. Como pode ser observado na Tabela 1, o sêmen não sexado do touro 1 apresentou na média geral valores maiores que os touros 2 e 3 quanto aos parâmetros da cinética espermática. É possível que estas características dos movimentos espermáticos (VCL, VSL, VAP, ALH e BCF) tenham contribuído para seu melhor desempenho na produção de embriões in vitro. Os parâmetros da cinética espermática têm sido positivamente correlacionados com fertilidade ${ }^{(19)}$, dentre esses parâmetros, o VCL, VSL e ALT já foram correlacionados à hiperatividade espermática, que é considerada um padrão de motilidade observado em espermatozóides que estejam no processo de capacitação $^{(20)}$, e a utilização do sêmen já em processo de capacitação pode ter favorecido à fertilização in vitro.

Ao se analisarem as amostras do sêmen sexado, o T1 obteve os melhores resultados para produção de blastocistos, diferindo do T2. Nas análises do CASA, essas diferenças foram expressas de forma ainda mais definida, distinguindo-o positivamente dos demais touros nas variáveis VCL, VSL, VAP, em que é possível observar claramente que o T1 foi superior aos demais e o T3 superior ao T2, confirmando os resultados obtidos com produção in vitro, fortalecendo e corroborando dados pulicados sobre o importância da qualidade do sêmen e de sua influência na fertilidade ${ }^{(3,5)}$.

A porcentagem de espermatozoides móveis demonstrada nas variáveis VCL, VAP, STR, BCF revela uma correlação significativa com a probabilidade de se alcançar a prenhez como publicado para fertilidade na espécie humana ${ }^{(11)}$.

Em estudo realizado com sêmen sexado criopreservado, observaram-se valores baixos para motilidade ${ }^{(21)}$, o que não foi observado no presente estudo já que os valores da cinética (VCL, VSL e VAP) do sêmen sexado do T1 $(117,7 \pm 1,6 \mu \mathrm{m} / \mathrm{s} ; 60,0 \pm 0,3 \mu \mathrm{m} / \mathrm{s} ; 73,6 \pm 0,4 \mu \mathrm{m} / \mathrm{s}$, respectivamente), T2 $(80,2 \pm 2,3 \mu \mathrm{m} / \mathrm{s} ; 47,0 \pm 2,0 \mu \mathrm{m} / \mathrm{s} ; \quad 57,7 \pm 0,9 \mu \mathrm{m} / \mathrm{s}$, respectivamente) e T3 $(86,4 \pm 5,7 \mu \mathrm{m} / \mathrm{s}$; $46,2 \pm 2,7 \mu \mathrm{m} / \mathrm{s} ; 53,8 \pm 2,8 \mu \mathrm{m} / \mathrm{s}$, respectivamente), foram superiores aos do sêmen convencional, 
porém não refletindo na produção in vitro.

No entanto, a integridade de membrana está entre as características físicas afetadas pela sexagem, provavelmente, devido ao estresse mecânico, nesse sentido, a redução da pressão durante o processo de sexagem aumentou a sobrevivência do espermatozoide sexado e, consequentemente, as taxas de fertilização.

Índices de fertilidade têm sido associados aos padrões de movimento espermático, tendo em vista diferenças significativas observadas no padrão de movimento exercido por espermatozoides que atingem altas e baixas taxas de fertilização ${ }^{(22)}$.

A variável frequência de batimento de cauda (BCF) diferiu significativamente entre os touros e evidenciou maior correlação quando comparado aos valores de VCL ao se utilizar sêmen sexado ( $\mathrm{r}$ $=0,80)$ do que quando correlacionado ao sêmen convencional $(\mathrm{r}=0,69)$. É sabido que o deslocamento lateral de cabeça já foi correlacionado com a fertilização, por indicar vigor de batimento flagelar junto com a frequência de rotação da célula, que são, provavelmente, importantes para a progressão de espermatozoides ${ }^{(24)}$. Os dados obtidos no presente estudo corroboram resultados observados em outro experimento, em que o autor observou que os espermatozoides sexados demonstraram a maioria dos parâmetros de motilidade superiores aos submetidos à técnica convencional e afirmou existir fortes indícios de que espermatozoides bovinos que passam pela técnica de citometria de fluxo entram em estado de hiperativação ${ }^{(25)}$.

A avaliação espermática com o auxilio das sondas fluorescentes demonstrou que o sêmen sexado do T1 apresentou menor porcentagem de membrana plasmática íntegra em comparação ao T2 e T3 (respectivamente, $38 \pm 2,7 ; 44,1 \pm 4,4 ; 53,8 \pm 1,8$ ), demonstrando importância estatística em relação ao T3 ( $\mathrm{P}<0,05)$, o que contradiz com os resultados da produção embrionária e o princípio da importância da integridade de membrana para obter bom resultado fertilizante ${ }^{(5)}$. Provavelmente por se tratar de produção in vitro em que não existem barreiras naturais a serem transpassadas, $o$ percentual de lesão na membrana, com essa intensidade, somada às outras características com valores superiores aos demais, não influenciou de forma negativa no seu desempenho.

Apesar de a utilização do sêmen sexado na FIV ter apresentado índice de produção embrionária menor (114 blastocistos), quando comparado com a produção embrionária alcançada pelo sêmen convencional (151 blastocistos), ela é viável quando se busca a produção de embriões do sexo feminino, que é o interesse na pecuária de leite.

Considerando-se que, no sêmen sexado, $90 \%{ }^{(26)}$ dos produtos obtidos são do sexo desejado, pode-se considerar para o presente trabalho 102 embriões do sexo feminino dos 114 produzidos, enquanto que com o uso de sêmen convencional seriam produzidos $60 \%{ }^{(27)}$ de embriões do sexo masculino ou seja, dos 151 blastocistos obtidos com o sêmen convencional, aproximadamente 61 embriões seriam do sexo feminino, o que aumentaria os custos com o descarte de machos ${ }^{(28)}$, tornando-se justificável o emprego do sêmen sexado para a obtenção de embriões em explorações leiteiras ${ }^{(3)}$.

\section{Conclusão}

No presente trabalho o sêmen sexado foi menos eficiente na produção de blastocistos quando comparado ao sêmen convencional. As análises do CASA bem como as sondas fluorescentes foram compatíveis com o potencial fertilizante das amostras de sêmen sexado e convencional em touros da raça 5/8 Girolando. O uso de sêmen sexado na FIV é viável diante do número de embriões obtidos. 


\section{Referências}

1. Alexandre A, Marinho C, Vitor Abreu V, Barros KM. Boletim Setorial do Agronegócio, Bovinocultura Leiteira [Internet] Recife: Sebrae; 2010 May [cited 2013 Oct 6]. Available from:https://www.yumpu.com/pt/document/view/16730575/boletim-setorial-do-agronegocio-bovinoculturaleiteira

2. Varago FC, Mendonça LF, Lagares MA. Produção in vitro de embriões bovinos: estado da arte e perspectiva de uma técnica em constante evolução. Rev. Bras. Reprod. Anim. 2008 Set 05;32(2):100-109.

3. Seidel GE Jr. Economics of selecting for sex: the most important genetic trait. Theriogenology, 2003 Jan 15;59(2):585-98.

4. Amann RP, Katz DF. Reflections on CASA after 25 Years. Journal of Andrology, 2004 May/June;25(3):317-325.

5. Graham JK, Mocé E. Fertility evaluation of frozen/thawed semen. Theriogenology. 2005;64(3):492-504.

6. Marques, CC, Baptista MC, Pereira RM, Vasques MI, Lopes da LFC, Horta AEM. Influência do sêmen de diferentes touros sobre as taxas de fertilização in vitro e desenvolvimento de embriões em co-cultura. Revista Portuguesa de Zootecnia. 1995 out 30;2(2):103-110.

7. Watanabe YF, Oliveira Filho de EB. Efeito de reprodutores, distribuídos em três grupos genéticos, na produção in vitrode embriões bovinos. ARS Veterinaria, 2000;16(1):22-27.

8. Blondin P, Beaulieu M, Fournier V, Morin N, Crawford L, Madan P, King WA. Analysis of bovine sexed sperm for IVF from sorting to the embryo. Theriogenology. 2009;71(1):30-38.

9. Rijsselaere T, Van Soom A, Tanghe S, Coryn M, Maes D, de Kruif A. New techniques for the assessment of canine semen quality: A review. Theriogenology. 2005; 64(3): 706-719.

10. Silva ECB, Cajueiro JFP, Silva SV, Soares PC, Guerra MMP. Effect of antioxidants resveratrol and quercetin on in vitro evaluation of frozen ram sperm. Theriogenology. 2012;77(8):1722-1726.

11. Larsen L, Scheike T, Jersen TK, Bonde JP, Ernst E, Hjollund NH, Zhou Y, Skakkebaek NE, Giwercman

A. Computer- assisted semen analysis parameters for fertility of men form the general population. Human Reproduction. 2000;15(7):1562-1567.

12. Arruda RP, Celeghini ECC, Alonso MA, Carvalho HF, Oliveira LZ, Nascimento J, Silva DF, Affonso FJ, Lemes KM, Jaimes JD. Métodos de avaliação da morfologia e função espermática: momento atual e desafios futuros.Rev. Bras. Reprod. Anim. 2011 abr./jun;35(2):145-151.

13. Samplaski MK, Agarwal A, Sharma R, Glickman ES. New generation of diagnostic tests for infertility: Review of specialized semen tests. International Journal of Urology. 2010;17(10):839-847.

14. Arruda RL, Orro IR, Passos TS, Costa e Silva EV, Zúccari CESN. Técnicas para avaliação laboratorial da integridade estrutural e funcional do sêmen congelado de touros. Rev. Bras. Reprod. Anim. 2010;34(3):168-184.

15. Gonçalves PBD, Figueiredo JR, Freitas VJF. Biotécnicas aplicadas à reprodução animal. 2a . ed., Roca, São Paulo, 395 p., 2008

16. Camargo LSA, Boite MC, Viana SW, Mota GB, Serapiao RV, Sa WF, Viana JHM, Nogueira LAG. Osmotic challenge and expression of aquaporin 3 and $\mathrm{Na} / \mathrm{K}$ ATPase genes in bovine embryos produced in vitro. Cryobiology. 2011;63(3):256-262. 
17. Silva SV, Soares AT, Batista AM, Almeida FC, Nunes JF, Peixoto CA, Guerra MMP. In vitro and in vivo evaluation of ram sperm frozen in Tris egg-yolk and supplemented with superoxide dismutase and reduced glutathione. Reprod. Domest. Anim.2011 Oct;46(5):874-81.

18. Guthrie HD, Welch GR. Determination of intracellular reactive oxygen species and high mitochondrial membrane potential in Percoll-treated viable boar sperm using fluorescence-activated flow cytometry. Journal Animal Science, 2006 Aug;84(8):2089-100.

19. Lavara R, Mocé E, Lavara F, Castro MPV, Vicente JS. Do parameters of seminal quality correlate with the result of on-farm inseminations in rabbits? Theriogenology. 2005;64(5):1130-1141.

20. Farrel PB, Presicce GA, Brockett CC, Foot RH. Quantification of bull sperm characteristics by computer-assisted sperm analysis (CASA) and the relationship to fertility. Theriogenology. 1998;49(4):871879.

21. Carvalho JO, Sartori R, Machado GM, Mourão GB, Dode MAN. Quality assessment of bovine cryopreserved sperm after sexing by flow cytometry and their use in in vitro embryo production. Theriogenology. 2010;74(9):1521-1530.

22. Verstegen J, Iguer-ouada M, Oclin K. Computer assisted semen analyzers in andrology research and veterinary practice. Theriogenology. 2002;57(1):149-179.

23. Zhang BR, Larsson B, Lundeheim N, Håård MG, Rodriguez-Martinez H. Prediction of bull fertility by combined in vitro assessments of frozen-thawed semen from young dairy bulls entering an IA-programe. International Journal of Andrology. 1999;22(4):253-260.

24. Jeulin C, Lewin LM, Chevrier C, Schoevaert-Brossault D. Changes in flagellar movement of rat spermatozoa along the length of the epididymis: Manual and computer-aided image analysis. Cell Motil Cytoskeleton. 1996;3(5):147- 161.

25. Gallego AM. Avaliação das características da motilidade (CASA), morfologia e funcionalidade da membrana plasmática (HOST) de espermatozóides bovinos sexados por citometria de fluxo [Dissertação]. [São Paulo]: Universidade de São Paulo, Faculdade de Medicina Veterinária e Zootecnia; 2010. 118 p. Available from: http://www.teses.usp.br/teses/disponiveis/10/10131/tde-01042011-143106/pt-br.php

26. Cran DG, Cochrane DJ, Johnson L, Wei H, Lu KH, Polge C. Separation of X and Y chromosome bearing bovine sperm by flow citometry for use in IVF. Theriogenology. 1994;41(1):183.

27. Machado GM, Carvalho JO, Siqueira Filho E, Caixeta ES, Franco MM, Rumpf R, Dode MAN. Effect of Percoll volume, duration and force of centrifugation, on in vitro production and sex ratio of bovine embryos. Theriogenology, 2009; May71(8):1289-1297.

28. Melz LJ. Custos de produção de gado bovino: revisão sob o enfoque da contabilidade de custos. Custos e @ gronegócio online. $2013 \quad$ - $\quad$ Jan/Mar;9(1):119-136. $\quad$ Disponível em <http://www.custoseagronegocioonline.com.br/numero1v9/Bovino.pdf $>$, acesso em fevereiro de 2015. 